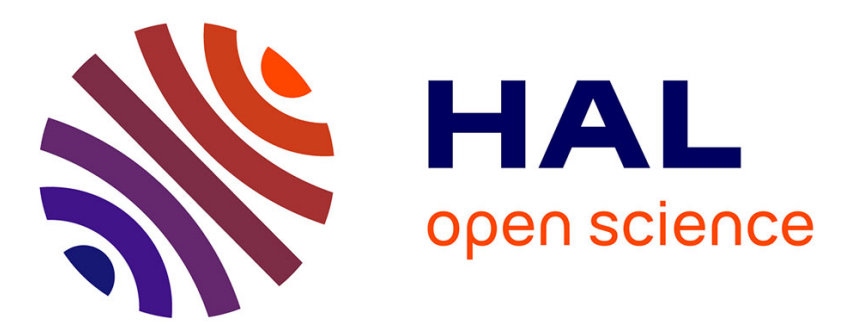

\title{
Mortality differences between the foreign-born and locally-born population in France (2004-2007).
}

Roxane Boulogne, Eric Jougla, Yves Breem, Anton E. Kunst, Grégoire Rey

\section{To cite this version:}

Roxane Boulogne, Eric Jougla, Yves Breem, Anton E. Kunst, Grégoire Rey. Mortality differences between the foreign-born and locally-born population in France (2004-2007).. Social Science and Medicine, 2012, 74 (8), pp.1213-23. 10.1016/j.socscimed.2012.01.002 . inserm-00835720

\section{HAL Id: inserm-00835720 https://www.hal.inserm.fr/inserm-00835720}

Submitted on 19 Jun 2013

HAL is a multi-disciplinary open access archive for the deposit and dissemination of scientific research documents, whether they are published or not. The documents may come from teaching and research institutions in France or abroad, or from public or private research centers.
L'archive ouverte pluridisciplinaire HAL, est destinée au dépôt et à la diffusion de documents scientifiques de niveau recherche, publiés ou non, émanant des établissements d'enseignement et de recherche français ou étrangers, des laboratoires publics ou privés. 


\section{Appendix}

Table A: Countries grouped on the basis of their 2004 Human Development Index score.

\begin{tabular}{|c|c|}
\hline Countries of birth groups & Countries of birth \\
\hline $\begin{array}{l}\text { High HDI countries } \\
\text { (greater than or equal to } 0.938 \text { ) }\end{array}$ & $\begin{array}{l}\text { Denmark, Iceland, Norway, Sweden, Finland, Austria, Italy, Belgium, the United Kingdom, Spain, Netherlands, Ireland } \\
\text { or Eire, Luxembourg, Switzerland, Japan, Canada, the United States, Australia. }\end{array}$ \\
\hline $\begin{array}{l}\text { Intermediate HDI countries } \\
\text { (greater than or equal to } 0.728 \text { and less than } 0.938 \text { ) }\end{array}$ & $\begin{array}{l}\text { Estonia, Latvia, Lithuania, Germany, Bulgaria, Hungary, Rumania, Rep Tchèque, Slovakia, Bosnia and Herzegovina, } \\
\text { Montenegro, Serbia and Montenegro, Poland, Russia, Albania, Greece, Portugal, Malta, Slovenia, Belarus, Ukraine, } \\
\text { Yugoslav Ex rep of Macedonia, Saudi Arabia, Iran, Lebanon, Israel, Turkey, Siberia, China, Thailand, the Filipinas, } \\
\text { Jordan, Singapore, Malaysia, Hong-Kong, Sri Lanka, Korea (rep of), Kuwait, Bahrain, Armenia, Azerbaijan, Cyprus, } \\
\text { Georgie, Kazakhstan, Palestine, Libya, Algeria, Maurice, Seychelles, Costa Rica, Cuba, Dominican ( Rep), Panama, El } \\
\text { Salvador, Argentina, Brazil, Chile, Colombia, Ecuador, Paraguay, Peru, Uruguay, Venezuela, Trinidad and Tobago, } \\
\text { Grenada, the Bahamas, Surinam, Dominique, Saint Lucie, New Zealand, East Samoa, Fiji, Mexico. }\end{array}$ \\
\hline $\begin{array}{l}\text { Low HDI countries } \\
\text { (less than } 0.728 \text { ) }\end{array}$ & $\begin{array}{l}\text { Moldavia, Iraq, Syria, Afghanistan, Pakistan, Nepal, India, Burma, Indonesia, Cambodia, Korea (Democratic popular } \\
\text { Rep of), Laos, Mongolia, Vietnam, Bangladesh, Yemen, Kirghizia, Uzbekistan, Tadjikistan, Turkmanistan, Egypt, } \\
\text { Liberia, South Africa, Gambia, Tanzania, Zimbabwe, Namibia, Congo ( democratic Rep), equatorial Guinea, Ethiopia, } \\
\text { Eritrea, Somalia, Burundi, Cameroon, the Central African Republic, Congo, Ivory Coast, Benin, Gabon, Ghana, Guinea, } \\
\text { Burkina, Kenya, Madagascar, Malawi, Mali, Mauritania, Niger, Nigeria, Uganda, Rwanda, Senegal, Sierra Leone, } \\
\text { Sudan, Chad, Togo, Zambia, Lesotho, Morocco, Tunisia, Swaziland, Guinea Bissau, Mozambique, Sao Tome e } \\
\text { Principe, Angola, Cape Verde, Comoros, Djibouti, Guatemala, Haiti, Honduras, Nicaragua, Bolivia, Jamaica, Vanuatu. }\end{array}$ \\
\hline
\end{tabular}


Table B: Relative risk of mortality for foreign-born populations relative to the locally-born population by specific causes of death and gender (years 2004-2007)

\begin{tabular}{|c|c|c|c|c|c|c|c|c|c|c|c|c|c|c|c|}
\hline \multirow[b]{3}{*}{ Country of birth } & \multirow{2}{*}{\multicolumn{12}{|c|}{ Violent death }} & \multirow{2}{*}{\multicolumn{3}{|c|}{$\begin{array}{c}\text { Infectious disease } \\
\text { AIDS } \\
\end{array}$}} \\
\hline & & & & & & & & & & & & & & & \\
\hline & $\begin{array}{c}\text { Both } \\
\text { genders }\end{array}$ & Males & Females & $\begin{array}{c}\text { Both } \\
\text { genders }\end{array}$ & Males & Females & $\begin{array}{c}\text { Both } \\
\text { genders }\end{array}$ & Males & Females & $\begin{array}{c}\text { Both } \\
\text { genders }\end{array}$ & Males & Females & $\begin{array}{c}\text { Both } \\
\text { genders }\end{array}$ & Males & Females \\
\hline Algeria & 1.23 & $1.55^{*}$ & 0.79 & 0.90 & $0.85^{*}$ & 0.96 & $0.46^{*}$ & $0.45^{*}$ & $0.48^{*}$ & $0.78^{*}$ & $0.84 *$ & $0.63^{*}$ & $1.77^{*}$ & $1.66^{*}$ & $2.25 *$ \\
\hline Morocco & 1.07 & 1.25 & 0.78 & $0.64 *$ & $0.64 *$ & $0.64 *$ & $0.40^{*}$ & $0.37 *$ & $0.48^{*}$ & 0.89 & 0.98 & $0.58 *$ & 0.91 & 0.93 & 0.82 \\
\hline Tunisia & 0.64 & 0.43 & 1.05 & 0.89 & $0.82 *$ & 0.97 & $0.36^{*}$ & $0.35 *$ & $0.43 *$ & $0.66^{*}$ & $0.72 *$ & $0.46^{*}$ & 1.50 & 1.45 & 1.60 \\
\hline AFFA & 1.16 & 1.29 & 0.95 & $0.70 *$ & $0.66 *$ & 0.79 & $0.37 *$ & $0.33^{*}$ & $0.54 *$ & $0.68 *$ & $0.62 *$ & 0.91 & $9.00 *$ & $6.21 *$ & $20.21 *$ \\
\hline Other African countries & 1.25 & 0.85 & 1.98 & 0.65 & $0.56^{*}$ & 0.77 & $0.26^{*}$ & $0.28^{*}$ & $0.18 *$ & 0.73 & 0.74 & 0.74 & $5.98 *$ & $3.83 *$ & $15.02 *$ \\
\hline Turkey & $1.90 *$ & $2.18^{*}$ & 1.31 & 0.95 & 0.85 & 1.11 & $0.30^{*}$ & $0.26^{*}$ & $0.45^{*}$ & 0.68 & $0.70 *$ & 0.58 & 0.28 & 0.25 & 0.41 \\
\hline Central Asia & 0.91 & 0.27 & 2.30 & 0.64 & $0.60 *$ & 0.70 & $0.34 *$ & $0.35^{*}$ & $0.31 *$ & $0.46 *$ & $0.46^{*}$ & 0.47 & 0.41 & 0.49 & - \\
\hline ASFA & 1.10 & 1.06 & 1.17 & 0.90 & 0.86 & 0.96 & $0.36^{*}$ & $0.28^{*}$ & $0.58 *$ & 0.71 & $0.58 *$ & 1.12 & 1.54 & 1.55 & 1.46 \\
\hline Other Asian countries & 0.56 & 0.32 & 0.90 & 0.67 & $0.60^{*}$ & 0.86 & $0.44 *$ & $0.40 *$ & $0.55^{*}$ & $0.26^{*}$ & $0.23 *$ & 0.35 & 0.46 & 0.48 & 0.42 \\
\hline North America & 0.56 & 0.96 & - & 0.86 & $0.79 *$ & 0.99 & $0.63^{*}$ & $0.49^{*}$ & 0.94 & 0.69 & $0.81 *$ & 0.36 & 1.17 & 0.76 & 2.57 \\
\hline $\begin{array}{l}\text { Other American-Oceanian } \\
\text { countries }\end{array}$ & 1.36 & 1.96 & 0.54 & 0.78 & $0.58^{*}$ & 1.06 & $0.59^{*}$ & $0.49^{*}$ & 0.81 & $0.44 *$ & $0.50^{*}$ & 0.29 & $4.98^{*}$ & $4.87^{*}$ & $5.55^{*}$ \\
\hline Eastern European & $2.21 *$ & $2.77 *$ & 1.61 & $1.19 *$ & $1.27 *$ & $1.14 *$ & $0.78^{*}$ & $0.78 *$ & 0.78 & 1.01 & 1.10 & 0.84 & 1.26 & 1.08 & 1.82 \\
\hline Other European countries & 0.88 & 1.01 & 0.71 & 0.95 & $0.90 *$ & 1.01 & $0.68^{*}$ & $0.68^{*}$ & $0.69^{*}$ & 0.88 & $0.87^{*}$ & 0.90 & 0.97 & 0.91 & 1.21 \\
\hline All foreign countries & 1.11 & 1.24 & 0.92 & 0.91 & $0.84 *$ & 0.98 & $0.51^{*}$ & $0.49^{*}$ & $0.57^{*}$ & $0.77 *$ & $0.80^{*}$ & $0.73^{*}$ & $2.12 *$ & $1.74 *$ & $3.66^{*}$ \\
\hline
\end{tabular}

*: RR is statistically significantly different from 1 , at the $5 \%$ level. 